\title{
Functional integrated modulators and receivers utilizing PLC hybrid integration technology for coherent transmission
}

\author{
Shinji Mino ${ }^{a)}$, Hiroshi Yamazaki, Takashi Goh, Ken Tsuzuki, \\ Takashi Saida, Takaharu Ohyama, Ikuo Ogawa, \\ and Koichi Murata \\ NTT Photonics Laboratories, NTT Corporation \\ 3-1 Morinosato Wakamiya, Atsugi-shi, Kanagawa, 243-0198, Japan \\ a)mino.shinji@lab.ntt.co.jp
}

\begin{abstract}
We review our recent progress toward transmission speeds per channel of $100 \mathrm{Gbit} / \mathrm{s}$ and beyond, focusing on optical modulators and receivers for coherent transmission using PLC integration technology. We have used PLC-LiNbO 3 hybrid integration technology to develop optical multilevel modulators, including a DP-QPSK modulator and a $64 \mathrm{QAM}$ modulator for $100 \mathrm{Gbit} / \mathrm{s}$ and post $100 \mathrm{Gbit} / \mathrm{s}$. This technology provides practical performance and scalability for various advanced modulations. We have also developed flexible-format modulators for future elastic networks, which will enable the efficient use of the spectral resource. This modulator can select an optimum carrier number and modulation level according to the transmission conditions. In terms of receivers, we have developed integrated optical receiver front-ends for $100 \mathrm{Gbit} / \mathrm{s}$ DP-QPSK based on hermetically sealed $\mathrm{O} / \mathrm{E}$ converters. PLC hybrid-integration technologies are promising for compact, practical, cost-effective, and highly reliable coherent optical modulators and receivers.
\end{abstract}

Keywords: Coherent, PLC, lithium niobate, modulator, receiver, integration

Classification: Optoelectronics, Lasers and quantum electronics, Ultrafast optics, Silicon photonics, Planar lightwave circuits

\section{References}

[1] D.-S. Ly-Gagnon, K. Katoh, and K. Kikuchi, "Unrepeated 210-km transmission with coherent detection and digital signal processing of 20-Gb/s QPSK signal," Proc. OFC, OThL4, 2005.

[2] Optical internetworking forum (OIF), "Implementation agreement for integrated polarization multiplexed quadrature modulated transmitters," [Online] http://www.oiforum.com/public/documents/OIF-PMQTX-01.0.pdf, [Online] OIF_DPC_RX-01.0.pdf 
[3] A. Sano, et al., "100 x 120-Gb/s PDM 64-QAM transmission over $160 \mathrm{~km}$ using linewidth-tolerant pilotless digital coherent detection," Proc. ECOC2010, paper PD2.4, 2010.

[4] S. Okamoto, et al., "512 QAM (54 Gbit/s) coherent optical transmission over $150 \mathrm{~km}$ with an optical bandwidth of $4.1 \mathrm{GHz}$," Proc. ECOC2010, paper PD2.3, 2010.

[5] A. Sano, et al., "69.1-Tb/s (432 x 171-Gb/s) C- and extended L-band transmission over $240 \mathrm{~km}$ using PDM-16-QAM Modulation and digital coherent detection," Proc. OFC/NFOEC2010, paper PDPB7, 2010.

[6] H. Masuda, et al., "13.5-Tb/s $(135 \times 111-\mathrm{Gb} / \mathrm{s} / \mathrm{ch})$ no-guard-interval coherent OFDM transmission over $6,248 \mathrm{~km}$ using SNR maximized secondorder DRA in extended L-band," Proc. OFC/NFOEC '09, paper PDPB5, 2009.

[7] T. Sakamoto, et al., "50-Gb/s 16 QAM by a quad-parallel Mach-Zehnder modulator," Proc. ECOC200\%, paper PDS2.8, 2007.

[8] C. R. Doerr, et al., "Monolithic InP 16-QAM modulator," Proc. OFC/NFOEC2008, paper PDP20, 2008.

[9] G. Lu, et al., "16-QAM transmitter using monolithically integrated quad Mach-Zehnder IQ modulator," Proc. ECOC2010, paper Mo.1.F.3, 2010.

[10] T. Yamada, et al., "Compact 111-Gb/s integrated RZ-DQPSK modulator using hybrid assembly technique with silica-based PLCs and $\mathrm{LiNbO}_{3}$ devices," Proc. OFC/NFOEC2008, paper OThC3.

[11] N. Kikuchi, et al., "Intersymbol interference (ISI) suppression technique for optical binary and multilevel signal generation," J. Lightw. Technol., vol. 25, pp. 2060-2068, 2007.

[12] H. Yamazaki, et al., "64 QAM modulator with a hybrid configuration of silica PLCs and $\mathrm{LiNbO}_{3}$ phase modulators," Photon. Technol. Lett., vol. 22, pp. 344-346, 2010.

[13] H. Yamazaki, et al., "Modulation-level-selectable optical modulator with a hybrid configuration of silica PLCs and $\mathrm{LiNbO}_{3}$ phase modulators," Proc. ECOC2010, paper We.8.E.1, 2010.

[14] T. Goh, et al., "Novel flexible-format optical modulator with selectable combinations of carrier numbers and modulation levels based on silicaPLC and $\mathrm{LiNbO}_{3}$ hybrid integration," Proc. OFC/NFOEC2011, paper OWV2, 2011.

[15] H. Takeuchi, et al., "Monolithic integrated coherent receiver on InP substrate," IEEE Photon. Technol. Lett., vol. 1, pp. 398-400, 1989.

[16] S. Chandrasekhar, et al., "Monolithic balanced p-i-n/HBT photoreceiver for coherent optical heterodyne communications," IEEE Photon. Technol. Lett., vol. 3, pp. 537-539, 1991.

[17] C. R. Doerr, et al., "Monolithic silicon coherent receiver," Proc. OFC 2009, PDPB2, 2009.

[18] C. R. Doerr, et al., "Monolithic InP Multiwavelength coherent receiver using a chirped arrayed waveguide grating," IEEE J. Lightw. Technol., vol. 29, pp. 536-541, 2011.

[19] V. Houtsma, et al., "Manufacturable monolithically integrated InP dualport coherent receiver for $100 \mathrm{G}$ PDM-QPSK application," Proc. OFC 2011, OML2, 2011.

[20] A. Matiss, et al., "Novel integrated coherent receiver module for $100 \mathrm{G}$ serial transmission," Proc. OFC 2010, PD-PB3, 2010.

[21] A. Beling, et al., "Fully-integrated polarization-diversity coherent receiver module for 100 G DP-QPSK," Proc. OFC 2011, OML5, 2011.

[22] A. Sano, et al., "240-Gb/s polarization-multiplexed 64-QAM modulation and blind detection using PLC-LN hybrid integrated modulator and 
digital coherent receiver," Proc. ECOC2009, paper PD2.2, 2009.

[23] H. Yamazaki, et al., "Integrated 100-Gb/s PDM-QPSK modulator using a hybrid assembly technique with silica-based PLCs and $\mathrm{LiNbO}_{3}$ phase modulators," Proc. ECOC2008, paper Mo.3.C.1, 2008.

[24] H. Takara, et al., "Distance-adaptive super-wavelength routing in elastic optical path network (SLICE) with optical OFDM," Proc. ECOC2010, paper We.8.D.2, 2010.

[25] T. Ohyama, et al., "All-in-one $100 \mathrm{Gbit} / \mathrm{s}$ DP-QPSK coherent receiver using novel PLC-based integration structure with low-loss and widetolerance multi-channel optical coupling," Proc. OECC 2010, PD-P6, 2010.

[26] I. Ogawa, et al., "Packaging technology for ultra-small variable optical attenuator multiplexer (V-AWG) with multichip PLC integration structure using chip-scale-package PD array," IEEE J. Sel. Topics Quantum Electron., vol. 12, pp. 1045-1053, 2006.

[27] Y. Sakamaki, et al., "Dual polarisation optical hybrid using silica-based planar lightwave circuit technology for digital coherent receiver," Electron. Lett., vol. 46, no. 1, pp. 58-60, 2010.

[28] Y. Nasu, T. Mizuno, R. Kasahara, and T. Saida, "Temperature insensitive and ultra wideband silica-based dual polarization optical hybrid for coherent receiver with highly symmetrical interferometer desgin," submitted to ECOC 2011.

[29] T. Yoshimatsu, et al., "Composite-field MIC-PDs for low-bias-voltage operation," Proc. IPRM 2010, ThA2-5, 2010.

[30] H. Fukuyama, et al., "Two-channel InP HBT differential automatic-gaincontrolled transimpedance amplifier IC for 43-Gbit/s DQPSK Photoreceiver," Proc. CSIC 2008, 2008.

\section{Introduction}

The rapid increase in data and video traffic has led to a strong demand for speeds of $100 \mathrm{Gbit} / \mathrm{s}$ per channel and beyond in the transport network. Digital coherent detection utilizing polarization division multiplexing such as dual-polarization quadrature-phase-shift keying (DP-QPSK) [1] has recently attracted great attention due to its ability to receive high spectral efficiency signals with high receiver sensitivity, and to compensate digitally for transmission impairments such as chromatic dispersion and polarization mode dispersion. Thus the research and development of coherent transmission systems has been extensively pursued throughout the world to realize a large capacity of $100 \mathrm{Gbit} / \mathrm{s}$ and more than $100 \mathrm{Gbit} / \mathrm{s}$ per channel.

Figure 1 (a) and (b), respectively, show typical block diagrams of a transmitter and a receiver for digital coherent transmission systems [2]. Compared with direct detection transmission systems including on-off-keying (OOK) and differential phase shift keying (DPSK), coherent transmission systems require highly-functional complicated optics on both the transmitter and receiver sides. As these devices will be installed along with digital signal processors (DSPs) in conventional $5 \times 7$-inch transponder modules, they must be compact. Moreover, the optical skew in both modulators and receivers 
(a) $\mathrm{Tx}$
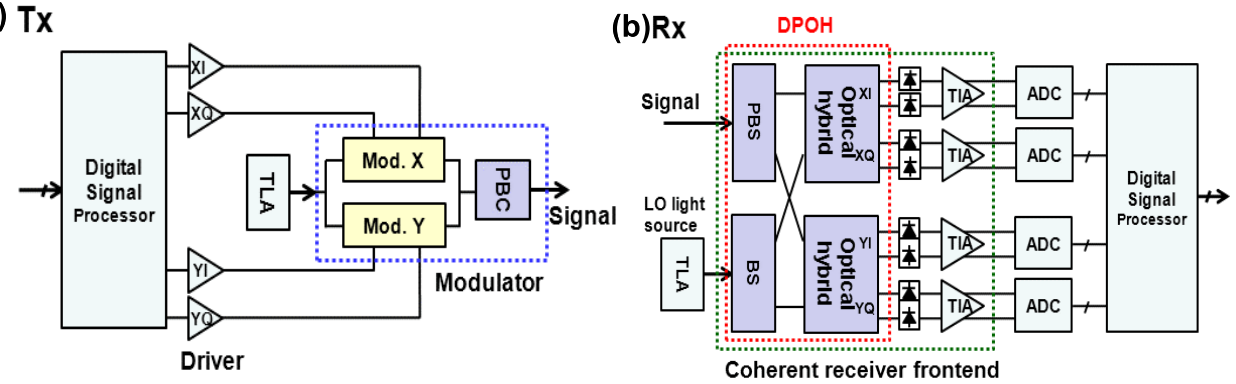

Fig. 1. Basic configurations of (a) transmitter and (b) receiver for $100 \mathrm{Gbit} / \mathrm{s}$ digital coherent transmission system.

must be precisely controlled to handle four parallel transmission signals (XI, XQ, YI, and YQ). Integrated modulators and receiver front-ends are of great interest as regards meeting these requirements. The Optical Internetworking Forum (OIF) has standardized the components indicated by dotted boxes in Fig. 1 (a) and (b). Furthermore, various advanced multilevel formats such as quadrature amplitude modulation (QAM) and orthogonal frequency-division multiplexing (OFDM) can also be used in digital coherent transmission systems.

As for the modulator, to achieve these advanced modulations, multilevel electronics such as arbitrary waveform generators (AWGs) or digitalto-analogue converters (DACs) have been used in many transmission experiments $[3,4]$. By using them, we can cover various modulation formats with a simple optical setup. On the other hand, optical multilevel-signal syntheses, in which only binary electronics is used, have also been studied extensively [5, 6, 7, 8, 9, 10, 11]. These schemes are promising for high-speed multilevel modulation because binary electronics poses fewer challenges for high-speed operation than multilevel electronics [8, 11].

Several integrated optical modulators for the optical syntheses have been reported. Modulators for 16 QAM [7] and 64 QAM [12] with parallel (MachZehnder modulator) MZM configurations have been demonstrated; both employ planar lightwave circuit (PLC)- $\mathrm{LiNbO}_{3}$ (LN) integration. Other configurations for 16 QAM have also been reported, such as a tandem/parallel MZM with LN monolithic integration [9], and a parallel electro-absorption modulator (EAM) with InP monolithic integration [8]. Flexible-format modulators utilizing PLC-LN integration have been developed for flexible optical networks, which enables efficient use of optical spectral resources [13, 14].

At the same time, integrated receiver front-ends for coherent detection have also been studied extensively using a number of approaches as summarized in Table I $[15,16,17,18,19,20,21,25]$. Various types of monolithic InP integrated receiver front-ends have been reported in a photonic integrated circuit (PIC) and module.

The monolithic approaches with InP can integrate the various devices including local oscillators (LOs), 90-degree optical hybrids (OHs), photodiodes (PD), and trans-impedance amplifiers (TIAs). The size and the elimination 
Table I. Reported integrated coherent receiver front-ends.

\begin{tabular}{|l|l|l|l|l|l|l|l|l|l|}
\hline Ref.) & $\begin{array}{l}\text { WDM } \\
\text { demux. }\end{array}$ & LO & PBS & BS & OH & PD & TIA & ADC & Note \\
\hline$[15]$ & - & $\ln \mathrm{P}$ & - & - & $\ln \mathrm{P}$ & $\ln \mathrm{P}$ & - & - & $\mathrm{PIC}$ \\
\hline$[16]$ & - & - & - & - & - & $\ln \mathrm{P}$ & $\ln \mathrm{P}$ & - & $\mathrm{PIC}$ \\
\hline$[17]$ & - & - & $\mathrm{Si}$ & $\mathrm{Si}$ & $\mathrm{Si}$ & $\mathrm{Ge}$ & - & - & $\mathrm{PIC}$ \\
\hline$[18]$ & $\ln \mathrm{P}$ & - & $\ln \mathrm{P}$ & $\ln \mathrm{P}$ & $\ln \mathrm{P}$ & $\ln \mathrm{P}$ & - & - & PIC \\
\hline$[19]$ & - & - & - & $\ln \mathrm{P}$ & $\ln \mathrm{P}$ & $\ln \mathrm{P}$ & - & - & PIC \\
\hline$[20]$ & - & - & - & - & $\ln \mathrm{P}$ & $\ln \mathrm{P}$ & $?$ & - & Module \\
\hline$[21]$ & - & - & Bulk & Bulk & $\ln \mathrm{P}$ & $\ln \mathrm{P}$ & $?$ & - & Module \\
\hline$[25]$ & - & - & Silica & Silica & Silica & $\ln \mathrm{P}$ & $\operatorname{In} \mathrm{P}$ & - & Module \\
\hline
\end{tabular}

of optical alignment of these approaches are attractive. However, a practical polarization beam splitter has not yet been achieved with InP waveguides. Monolithic approaches with silicon photonics are also attractive [17]. The coupling loss between optical fiber and silicon waveguides and dark current at photodiode seem to have room for improvement. We believe that PLC hybrid integration is a practical approach that exploits the most suitable devices and integrates them.

We have worked on integrated modulators and receivers utilizing PLC hybrid integration technology. This paper introduces our activities in relation to integrated devices for digital coherent transmission systems including PLC-LN hybrid modulators for advanced modulation formats, and integrated coherent receivers utilizing PLC hybrid integration with hermetically sealed $\mathrm{O} / \mathrm{E}$ converters.

\section{PLC-lithium niobate hybrid modulators}

\subsection{Basic concept}

Figure 2 shows the basic structure of a PLC-LN hybrid integrated modulator. We use an LN chip with an array of simple straight phase modulators and PLCs that incorporate all the other circuit components, such as couplers and filters. This structure combines the large electro-optic bandwidth of LN and the excellent transparency and design flexibility of PLCs. Another merit is that this configuration is highly scalable because we can increase the level of integration by increasing the number of phase modulators in the LN chip and devising PLCs with corresponding complexity. As shown in Fig. 3, we

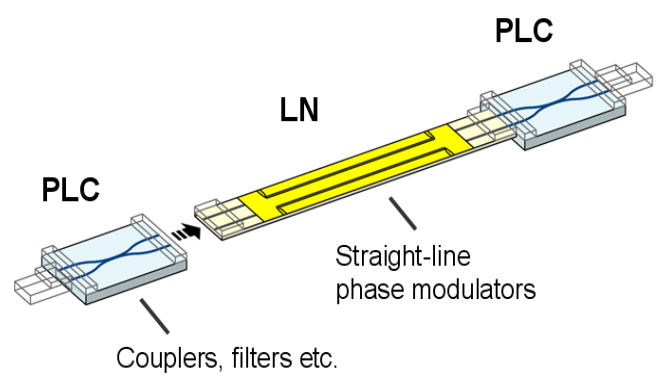

Fig. 2. Basic PLC-LN hybrid-integrated structure. 


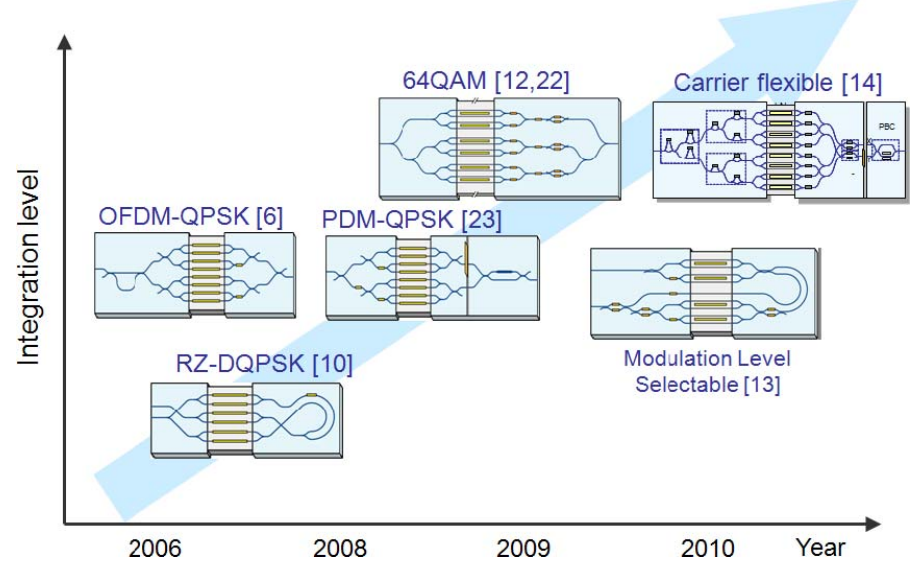

Fig. 3. PLC-LN hybrid modulators.

have developed various modulators with increasing levels of integration.

\subsection{DP-QPSK modulator}

The DP-QPSK modulator has the configuration shown in Fig. 4. Eight straight phase modulators with four high-speed signal electrodes in a Z-cut LN chip and thirteen couplers in PLCs make up two QPSK modulator circuits connected in parallel. In addition, a polarization multiplexing circuit, consisting of a polarization rotator with a half-wavelength plate (HWP), and a waveguide polarization beam combiner $(\mathrm{PBC})$ are connected to their output. Figure 5 shows a photograph of this module. The module package is $118 \times 13.5 \times 7 \mathrm{~mm}$, and $131 \mathrm{~mm}$ long when including both side fiber boots, which approaches the smallest size ever reported.

Typical characteristics of a $100 \mathrm{Gbit} / \mathrm{s}$ DP-QPSK modulator are listed in Table II. The overall optical insertion loss is less than $9 \mathrm{~dB}$ at a wavelength of $1.55 \mu \mathrm{m}$ including a polarization division intrinsic loss of $3 \mathrm{~dB}$.

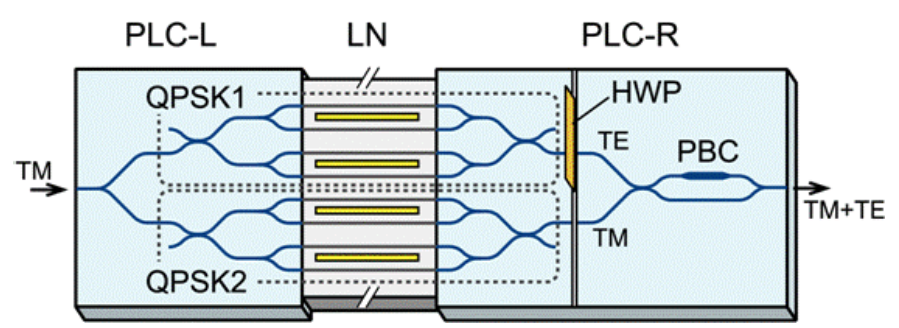

Fig. 4. Configuration of PLC-LN DP-QPSK modulator.

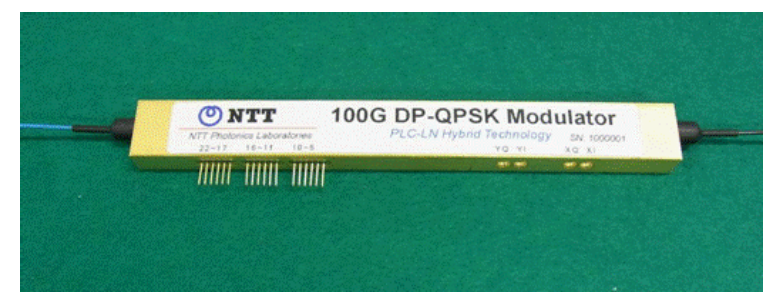

Fig. 5. Photograph of PLC-LN DP-QPSK modulator $(118 \times 16 \times 7 \mathrm{~mm})$ 
Table II. Typical properties and target specifications of a 100 G PDM-QPSK modulator.

\begin{tabular}{l|c|l}
\hline \multicolumn{1}{c|}{ Parameter } & Properties & Target spec. \\
\hline Insertion loss & $8.7 \mathrm{~dB}$ & $<14 \mathrm{~dB}$ \\
PDL & $0.1 \mathrm{~dB}$ & $<1.5 \mathrm{~dB}$ \\
Optical return loss & $>35 \mathrm{~dB}$ & $>30 \mathrm{~dB}$ \\
Extinction ratio; Parent MZI & $>46 \mathrm{~dB}$ & $>22 \mathrm{~dB}$ \\
\multicolumn{1}{c}{ Child MZI } & $>25 \mathrm{~dB}$ & $>20 \mathrm{~dB}$ \\
Polarization extinction ratio & $31 \mathrm{~dB}$ & $>20 \mathrm{~dB}$ \\
EO bandwidth & $>27 \mathrm{GHz}$ & $>23 \mathrm{GHz}$ \\
RF port $\mathrm{V} \pi @ 32 \mathrm{Gbaud}$ & $<3.5 \mathrm{~V}$ & $<3.5 \mathrm{~V}$ \\
\hline
\end{tabular}

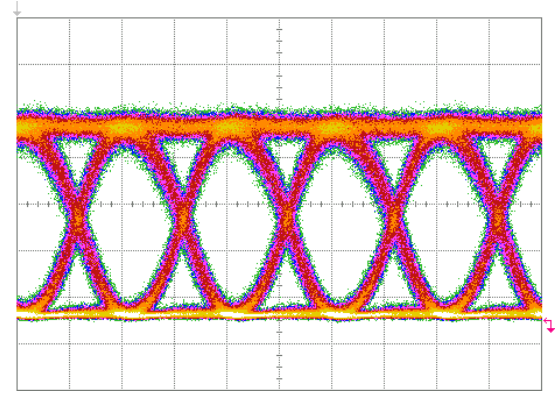

Fig. 6. Measured eye pattern.

The modulator has an electro-optic 3-dB bandwidth of $>27 \mathrm{GHz}$. The $\mathrm{RF}$ driving voltage was less than $3.5 \mathrm{~V}$ at $32 \mathrm{Gbit} / \mathrm{s}$. Figure 6 shows the measured eye pattern for a driving voltage of $3.0 \mathrm{~V}$. The modulator was driven with 32-Gbit/s non-return-zero $2^{31}-1$ pseudo-random bit sequences (PRBSs) in a back-to-back setup. Clear eye opening was obtained in this experiment. The OIF standardized a 100-Gbit/s integrated modulator in April 2010 [2]. Our DP-QPSK modulator complies with the target specifications suggested by the OIF.

\subsection{QAM modulator}

Figure 7 shows the configuration of a $64 \mathrm{QAM}$ modulator $[12,22]$. Three QPSK modulation circuits, each consisting of dual-parallel Mach-Zehnder modulators (MZMs), are connected in parallel by a pair of PLC asymmetric $1 \times 3 / 3 \times 1$ splitter/combiners, each with a power splitting/combining ratio

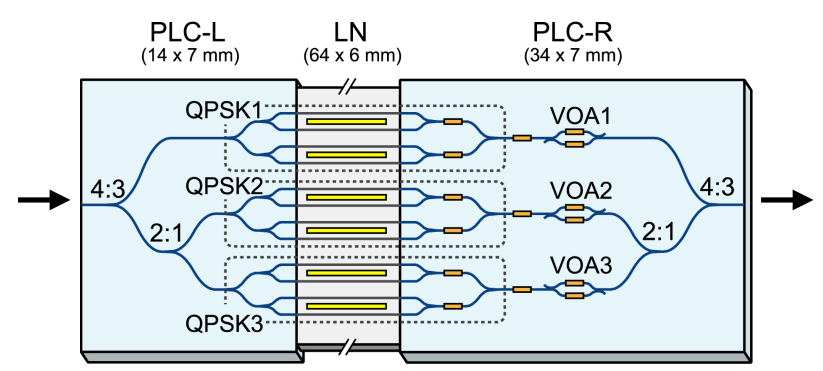

Fig. 7. Configuration of 64 QAM modulator. 


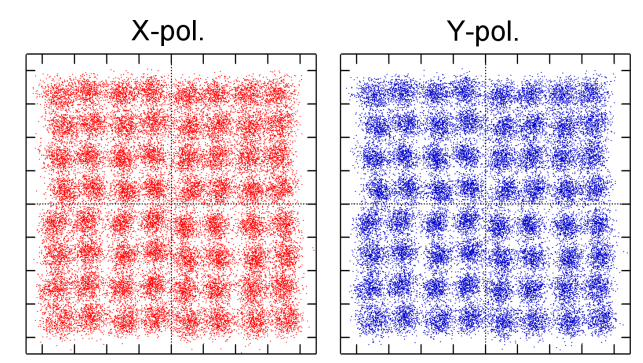

Fig. 8. Constellations of $240 \mathrm{Gbit} / \mathrm{s}$.

of $4: 2: 1$. These asymmetric circuits are designed by using the wavefront matching method (WFM), with which we can optimize the waveguide pattern on the basis of the desired splitting/combining ratio. PLC variable optical attenuators (VOAs) enable us to fine tune the power ratio. The 64 QAM signal is synthesized by coupling three QPSK signals with a field-amplitude ratio of $4: 2: 1$ (power ratio of $16: 4: 1$ ). The module has a small overall optical insertion loss of $5.5 \mathrm{~dB}$, as well as a broad electro-optic bandwidth of $>25 \mathrm{GHz}$.

By driving the modulator with six 20-Gbaud binary data signals and using an external DP circuit, we generated a 240-Gbit/s DP-64 QAM signal. The signal was received with a coherent receiver using a pilotless demodulation algorithm in an offline digital signal processor (DSP). Figure 8 shows constellations obtained with a back-to-back intradyne setup. The 64 signal points are clearly distinguished. The bit error rate was better than $1.3 \times 10^{-2}$.

\subsection{Flexible-format modulator}

The concept of a flexible optical network, in which various modulation formats are used flexibly to exploit spectral resources efficiently, has been the focus of attention in recent years [24]. We devised a flexible-format optical modulator with which we can select the optimum combinations of carrier number and modulation level according to the transmission conditions [14].

Figure 9 shows the configuration of a flexible-format optical modulator. The modulator circuit includes three tunable interleave filters (TILFs), eight parallel MZMs, two variable couplers (VCs), a polarization rotator with a HWP, and a waveguide PBC as shown in Fig. 9. TILFs and VCs are controlled with a PLC thermo-optic phase shifters. By tuning these TILFs and

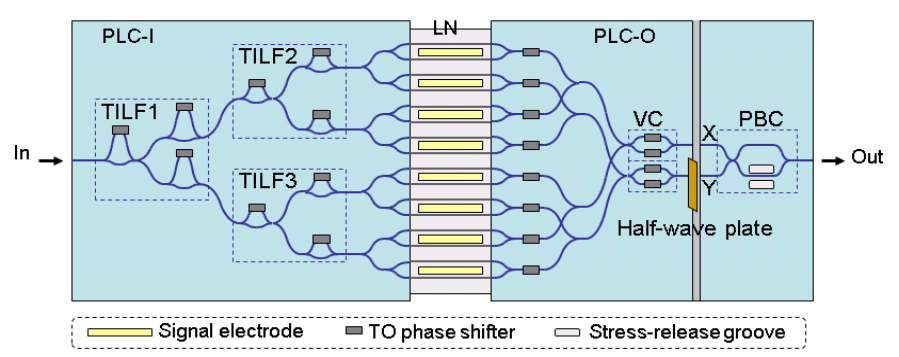

Fig. 9. Configuration of a flexible-format optical modulator [14]. 


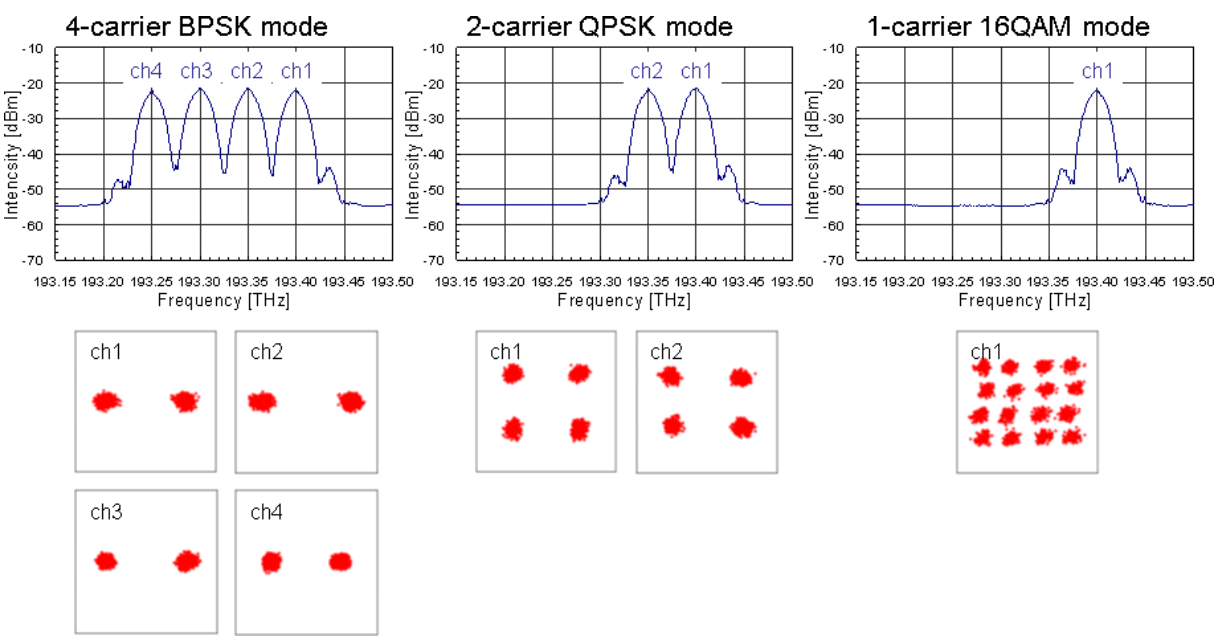

Fig. 10. Measured optical output spectra and constellations for 200-Gpbs operation in 4-carrier BPSK, 2-carrier QPSK, and 1-carrier 16-QAM modes.

VCs, we can flexibly change the combination of carrier number and modulation level according to the transmission conditions, such as the required transmission distance. Detailed principle is described in [14].

Figure 10 shows the measured optical output spectra and constellations of X-polarization signals. We also obtained the same clear constellation in Y-polarization signals. We demonstrated 4-carrier BPSK, 2-carrier QPSK, and 1-carrier 16-QAM operations, all with a bit rate of $200 \mathrm{Gbit} / \mathrm{s}$ and a symbol rate of 25 Gbaud, in total.

\section{Integrated coherent receiver front-ends [25]}

We have also worked on PLC hybrid integration technology to realize a coherent receiver front-end in a practical method. Figure 11 shows a schematic of our proposed configuration for an all-in-one DP-QPSK coherent receiver front-end. The receiver front-end consists of a PLC-based DPOH and a chip-scale packaged O/E converter (CSP-O/E) [26] with InP-based PDs and

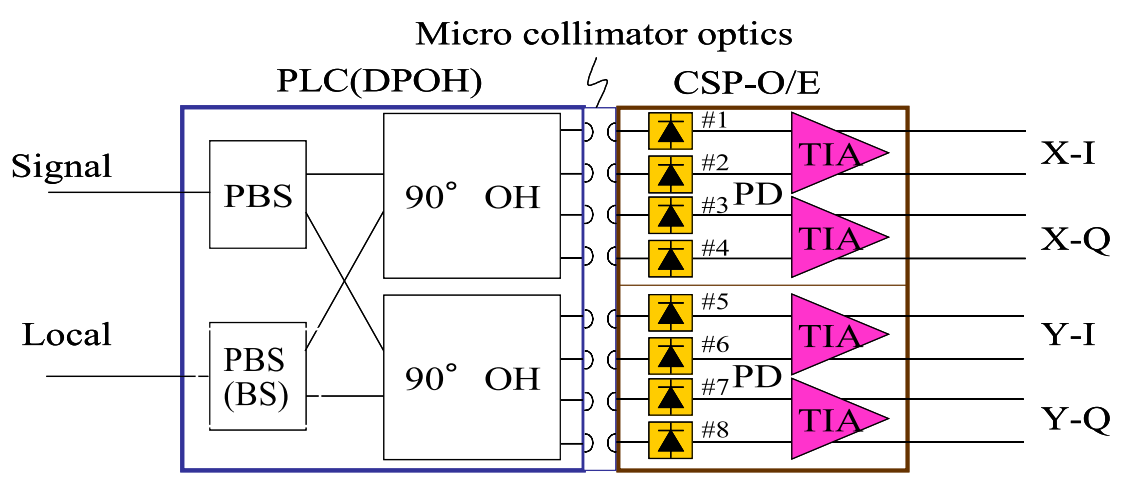

Fig. 11. DP-QPSK coherent receiver configuration using one-chip DPOH with PBSs and $90^{\circ} \mathrm{OHs}$, local hermetically sealed CSP-O/E with PDs and TIAs, and multi-channel micro collimator optics. 
(a)

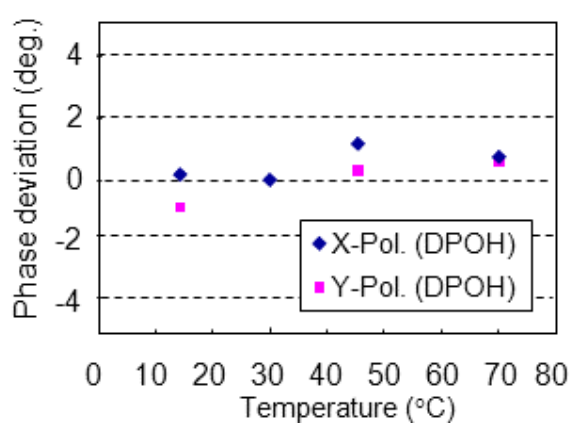

(b)

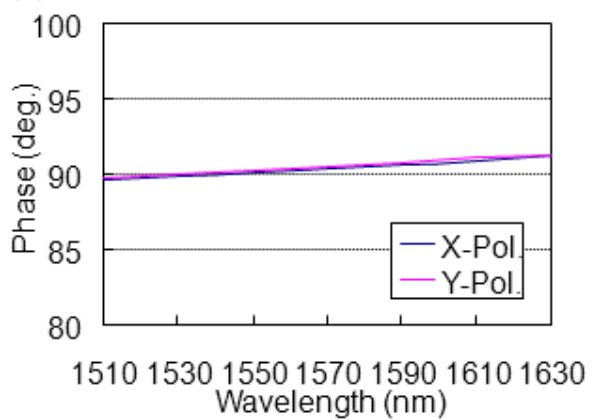

Fig. 12. (a) Temperature dependence and (b) wavelength dependence of PLC-based DPOH.

InP-based TIAs. Novel micro collimator optics was introduced to connect them optically.

The PLC-based DPOH is suitable for realizing stable and compact optical circuits for the coherent receiver. We modified the design of the DPOH for hybrid integration. The input signal is split into two orthogonal polarization components at a PBS, which consists of a symmetrical Mach Zehnder interferometer with polyimide quarter waveplates tilted at 0 and 90 degrees, respectively, inserted in the two arms. This configuration is highly symmetric and thus can suppress the temperature dependence of the polarization extinction ratio (PER). A polyimide half waveplate is inserted after one output port of the PBS to rotate the polarization. Figure 12 shows an example of the reported optical performance of the PLC-based DPOH for the coherent receiver. This $\mathrm{DPOH}$ exhibits a low phase deviation from 90 degrees of less than 3 degrees taking account of the temperature and wavelength dependence [27]. Furthermore, the high PER is better than $25 \mathrm{~dB}$ over the C-band. This PLC-based DPOH was fabricated with a refractive index difference of $1.5 \%$ and a core size of $4.5 \times 4.5 \mu \mathrm{m}[27,28]$. The chip size is $22 \times 16 \mathrm{~mm}$.

We developed a new compact $\mathrm{O} / \mathrm{E}$ assembly structure suitable for micro collimator optics. To maintain the reliability of the PD and TIA, we developed the CSP-O/E shown in Fig. 13. The CSP-O/E consists of a very small ceramic package with heatsink and a window in which the PD array chip [29] and the TIA array chip [30] are hermetically sealed. The signal output of the ceramic package has a 3 - $\mathrm{dB}$ bandwidth of over $40 \mathrm{GHz}$, which is wide enough to handle 32-Gbaud electrical signals from the TIA. The CSP-O/E size is $9.2 \times 8.2 \times 1.4 \mathrm{~mm}$. A flexible print circuit $(\mathrm{FPC})$ with DC block capacitors is attached to the $\mathrm{CSP}-\mathrm{O} / \mathrm{E}$. In the -5 to $80^{\circ} \mathrm{C}$ temperature range, we expect the relative positions of the $\mathrm{DPOH}$ and the PDs in CSP-O/E to vary by around $\pm 5 \mu \mathrm{m}$ in the $\mathrm{X}$ and $\mathrm{Y}$ directions. This variation may cause an optical coupling variation, which will result in a $\mathrm{PD}$ responsivity imbalance. Therefore, the optical connection between the DPOH and the CSP-O/E requires microminiaturized optics with a low loss and a wide coupling tolerance.

For this purpose, we introduced multi-channel micro collimator optics as shown in Fig. 13. The collimator optics with $45^{\circ}$ mirrors consists of a 1st 


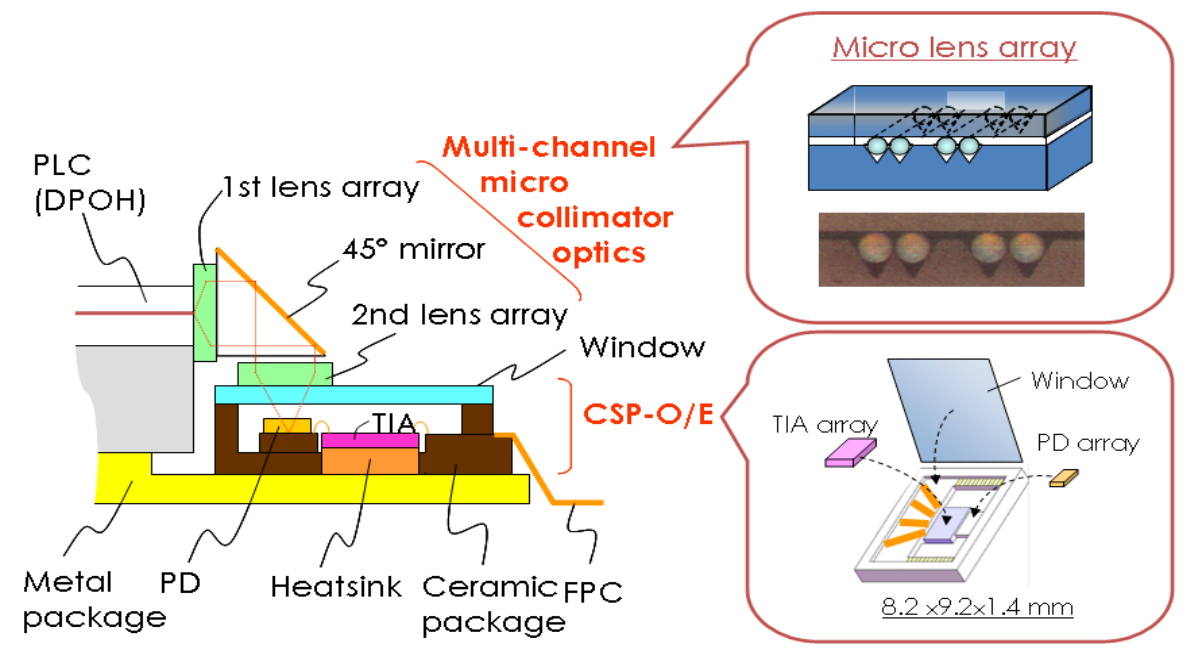

Fig. 13. Cross-sectional view of integration structure.

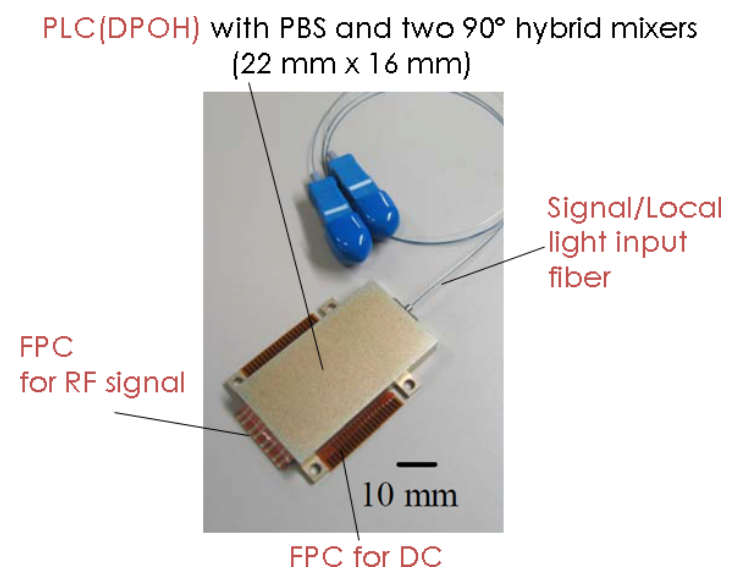

Fig. 14. Photograph of fabricated all-in-one DP-QPSK coherent receiver. Module size is $50 \times 27 \times 6 \mathrm{~mm}$.

lens array attached to a PLC and a 2nd lens array attached to the window of the CSP-O/E. We designed the collimation path via the mirror to be $2.5 \mathrm{~mm}$ long. The lens array is composed of graded index (GRIN) optical fibers. We measured the coupling tolerance between the PLC waveguide and a PD with a diameter of $19 \mu \mathrm{m}$, and confirmed that the micro collimator optics had a very wide tolerance of over $\pm 10 \mu \mathrm{m}$ at a $0.2-\mathrm{dB}$ down width. This result shows that the micro optics has sufficient tolerance to allow a coupling deviation caused by a temperature change. The photograph in Fig. 14 shows a fabricated DPQPSK coherent receiver front-end. Two CSP-O/Es are optically aligned with the PLC through the micro collimator optics. The module size is $27 \mathrm{~mm} \times$ $50 \mathrm{~mm} \times 6 \mathrm{~mm}$.

Figure 15 shows the responsivity of the receiver. The responsivity includes the $\mathrm{DPOH}$ insertion loss of $10.8 \mathrm{~dB}$ of which the inherent splitting loss is $9 \mathrm{~dB}$. The excess loss of the micro collimator optics is around $0.4 \mathrm{~dB}$, and the responsivity imbalance between adjacent PDs is less than $0.4 \mathrm{~dB}$. Figure 15 (b) shows the responsivity variation in an environmental temperature range of -5 to $80^{\circ} \mathrm{C}$. We confirmed a variation of less than $\pm 0.25 \mathrm{~dB}$ for all $\mathrm{PD}$ 

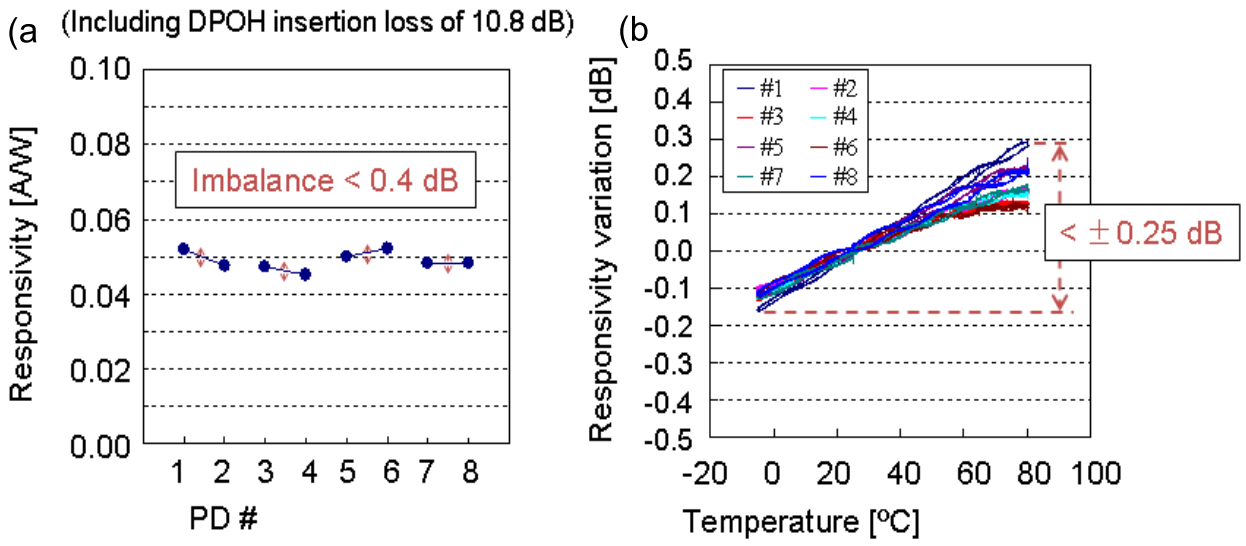

Fig. 15. Optical characteristics of receiver module (a) PD responsivity including the PLC-based DPOH. (b) $\mathrm{PD}$ responsivity deviation against temperature change of $-5 \mathrm{deg}$. to $80 \mathrm{deg}$. The deviation is within $\pm 0.25 \mathrm{~dB}$.
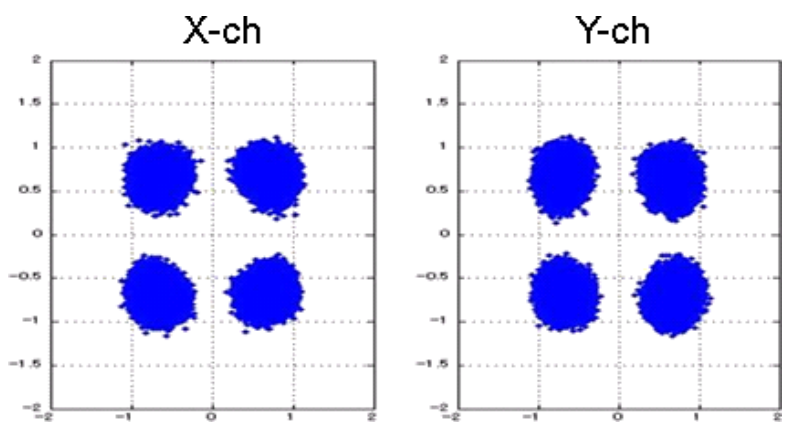

Fig. 16. Measured constellation diagrams at 112-Gbit/s DP-QPSK back-to-back detection.

channels. These results show that the micro optics have a stable structure in practical use, because a responsivity variation of less than $0.8 \mathrm{~dB}$ between PDs is required for 100-Gbit/s DP-QPSK signal detection when we assume a skew of \pm 1 ps.

We evaluated the performance of the receiver with $112 \mathrm{Gbit} / \mathrm{s}$ DP-QPSK (28-Gbaud x 2-digit x 2-polarizations) signals. Figure 16 shows the resulting constellation diagrams under a back-to-back condition at a signal light power of $-10 \mathrm{dBm}$ and a local light power of $4 \mathrm{dBm}$. A good separated constellation was observed for both polarization channels.

\section{Conclusion}

We have developed highly functional modulators and a compact integrated optical front-end utilizing PLC hybrid integration technology for $100 \mathrm{Gbit} / \mathrm{s}$ and post-100 Gbit/s coherent transmission systems. We have successfully demonstrated a DP-QPSK modulator by using PLC-LN integration technology. This technology is also scalable toward beyond $100 \mathrm{Gbit} / \mathrm{s}$, including 64 QAM modulators and OFDM modulator. Furthermore we have successfully demonstrated flexible modulators with which we can select the optimum 
combination of carrier number and modulation level for future efficient flexible networks. In terms of receivers, we have successfully demonstrated an integrated optical front-end for $100 \mathrm{Gbit} / \mathrm{s}$ DP-QPSK by using our newly developed silica-based PLC hybrid integration technologies with multi-channel micro collimator optics and hermetically sealed O/E converters. PLC integration technologies can be applied to compact, cost-effective, and highly reliable coherent optical modulators and receivers.

\section{Acknowledgments}

We would like to thank T. Yamada, Y. Doi, M. Ishii, H. Tanobe, R. Kasahara, S. Tsunashima, Y. Sakamaki, Y. Nasu, T. Yoshimatsu, H. Fukuyama for technical support and fruitful discussions, A. Kaneko, S. Suzuki for their guidance and encouragement, and H. Kawakami, E Yoshida, A Sano, and Y. Miyamoto for applications to system experiments.

This work is supported in part by the R\&D on "High-speed Optical Transport System Technologies" and "High-speed Optical Edge Node Technologies" of the Ministry of Internal Affairs and Communications (MIC) of Japan.

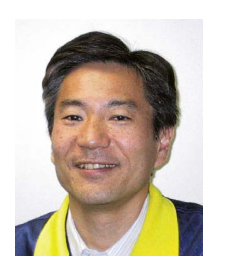

\section{Shinji Mino}

received a B.Sc. degree in chemistry from Waseda University in 1986, and an M.Sc., and Ph.D. in chemistry and electronic engineering from Tokyo University in 1988 and 1996, respectively.

In 1988, he joined NTT Opto-Electronics Laboratories, (now NTT Photonics Laboratories), Japan. He has been engaged in the research and development of the hybrid-integration technology of silica-based PLCs and various active devices such as $\mathrm{LDs}$ and $\mathrm{PDs}, \mathrm{LiNbO}_{3}$ modulators, liquid crystal devices, and electronic ICs. His research interests include various types of high-speed and functional photonic components utilizing PLC hybrid integration.

Dr. Mino is a Group Leader in the Photonics Integration Laboratory, NTT Photonics Laboratories, NTT Corporation. He is a senior member of the IEEE LEOS and the IEICE, and a member of the Japan Society of Applied Physics (JSAP).

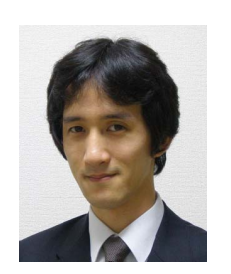

\section{Hiroshi Yamazaki}

received B.S. degree in integrated human studies in 2003 and the M.S. degree in human and environmental studies in 2005, both from Kyoto University, Kyoto, Japan. In 2005, he joined NTT Photonics Laboratories, Kanagawa, Japan, where he has been engaged in research on optical waveguide devices for communication systems. His current research interests include devices and systems for optical transmission using advanced multilevel modulation formats. Mr. Yamazaki is a member of Institute of Electronics, Information and Communication Engineers of Japan (IEICE). 


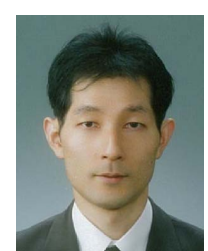

Takashi Goh

received the B.S. M.S. degrees in electronic and communication engineering from Waseda University, Tokyo, Japan, in 1991 and 1993, respectively. In 1993, he joined the NTT Opto-electronics Laboratories, Ibaraki (now NTT Photonics Laboratories, Kanagawa), Japan, where he has been engaged in research on planar lightwave circuit devices for communication. During 2002.2004, he was engaged in development on photonic network systems such as ROADM ring systems in NTT Innovation Laboratories. Currently, he is interested in devives for optical transmission using advanced modulation formats. Mr. Goh is a member of the Institute of Electronics, Information, and Communication Engineers of Japan (IEICE), the Japan Society of Applied Physics, and the IEEE.

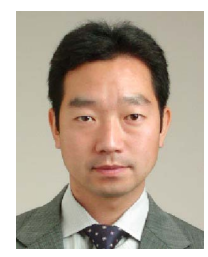

\section{Ken Tsuzuki}

received the B.S., M.S. and Ph.D. degrees in applied physics from Tohoku University in 1993, 1995 and 2006. In 1995 he joined NTT Optoelectronics Laboratories. Since then, he has been engaging in research and development on semiconductor photonic devices for optical fiber communication systems. From 2001 to 2002 he joined NTT Electronics Corporation. Now he is Senior Research Engineer in NTT Photonics Laboratories and engages in research and development on semiconductor photonic devices. Dr. Tsuzuki is a member of the Japan Society of Applied Physics and IEEE/LEOS.

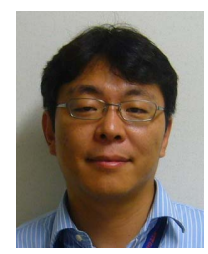

\section{Takashi Saida}

received B.S., and M.S. degrees, and a Ph.D. in Electrical Engineering in 1993, 1995, and 1998, respectively. After his joining to NTT OptoElectronics Laboratories, Ibaraki, Japan, in 1998, he began research on silica-based planar lightwave circuits (PLCs). From 2002 to 2003, He was a visiting scholar at Ginzton laboratory, tanford University, Stanford, CA. From 2006 to 2008, he was with NTT Electronics (NEL) on leave from NTT laboratories. His current interest is in high speed modulators and receivers for high speed optical transport systems. He is a member of IEICE, JSAP, IEEE and OSA.

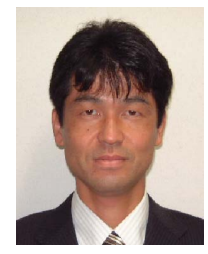

Takaharu Ohyama received the B.E. degree from Kyushu Institute of Technology, Fukuoka, in 1992 and the M.E. degree from Kyushu University, Fukuoka, in 1994. Since joining NTT Opto-electoronics Laboratories, Ibaraki, in 1994, he has been engaged in research on hybrid integration in silica-based PLCs and semiconductor devices. Now Mr. Ohyama is a Senior Research Engineer, Photonics Integration Laboratory, NTT Photonics Laboratories.a member of IEICE.

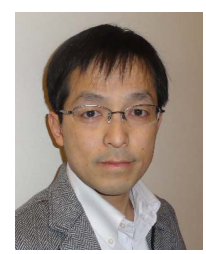

Ikuo Ogawa received the B.S. and M.S. degrees in applied physics from Waseda University, Tokyo, in 1990 and 1992, respectively. In 1992, he joined NTT Opto-electronics Laboratories. Since then, he has been engaged in R\&D of silica-based PLCs and hybrid integrated optical devices. Now he is Senior Research Engineer, Photonics Integration Laboratory, NTT Photonics Laboratories. Mr. Ogawa is a member of the Institute of Electronics, Information and Communication Engineers (IEICE), the Japan Society of Applied Physics (JSAP), and the IEEE Photonics Society. 


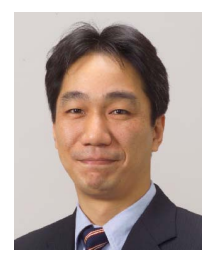

\section{Koichi Murata}

received the B.S. and M.S. degrees in mechanical engineering and the Dr. Eng. Degree in electrical and electronics engineering from Nagoya University, Nagoya, Japan, in 1987 and 1989, and 2003, respectively. In 1989 he joined NTT LSI Laboratories, Atsugi, Japan. He is currently a senior research engineer, supervisor at NTT Photonics Laboratories, Atsugi, Japan. He has been engaged in research and development of ultra-high speed mixed-signal ICs for optical communication systems. His current research interest includes optoelectronic IC design and highspeed optical transmission systems. Dr. Murata is a member of IEEE and IEICE of Japan. 